\title{
Absorption of ampicillin from the human lung
}

\author{
J. L. M A D D O C K S ${ }^{1}$ \\ Department of Bacteriology, Institute of Diseases of the Chest, Brompton, London
}

\begin{abstract}
Maddocks, J. L. (1975). Thorax, 30, 68-71. Absorption of ampicillin from the human lung. The absorption of ampicillin from the lungs after intratracheal administration was studied in a healthy human. Doses of $50 \mathrm{mg}, 250 \mathrm{mg}$, and $1,250 \mathrm{mg}$ were given by intratracheal injection, and absorption of ampicillin from the lungs was assessed by measuring plasma levels, the area under the plasma level-time curve, and urinary excretion. All these indices of absorption increased with the dose of ampicillin. An intratracheal dose of $100 \mathrm{mg}$ of probenecid together with $250 \mathrm{mg}$ of ampicillin failed to reduce the absorption of ampicillin from the lungs. The uptake of ampicillin by human foetal bronchial slices in vitro was also studied; although viable, they did not accumulate ampicillin. These preliminary results suggest that ampicillin is absorbed from the lungs by passive diffusion.
\end{abstract}

Relapse of bacterial infection is a major problem in the chemotherapy of chronic bronchitis (May, 1968). Haemophilus influenzae, the important pathogen, is only moderately sensitive to antibacterial drugs, most of which penetrate into bronchial secretions poorly and probably cause the bacteria to be only partially suppressed. An antibiotic often used for the treatment of bronchial infection is ampicillin. Although it is bactericidal against $H$. influenzae in vitro, it is only occasionally able to prevent relapse of $H$. influenzae bronchial infections and even then it has to be given in large doses (May and Delves, 1965). These authors consider this is due to the poor penetration of ampicillin through the blood-bronchial fluid barrier so that bactericidal levels for $H$. influenzae are not reached in respiratory tract secretions. Low levels of ampicillin have also been found in cerebrospinal fluid (Taber, Yow, and Nieberg, 1967) and aqueous humour (Goldman, Broughton, Javed, and Lauderdale, 1973) even when large parenteral doses are administered. These observations are in keeping with the physicochemical properties of ampicillin. It has an even stronger acidic group $(\mathrm{pKa}=2.52)$ than benzylpenicillin ( $\mathrm{pKa}=2 \cdot 72$ ) (Rapson and Bird, 1963) so that at blood $\mathrm{pH}$ it would be virtually completely ionized and therefore would be expected to pass through lipoid membrane barriers poorly. Another possible factor which might result Present address: KRUF Institute of Renal Disease, Cardiff Royal
Infirmary in low concentrations of ampicillin in bronchialo secretions is an active transport mechanism which pumps the drug out of bronchial fluid. Such a mechanism has been demonstrated for the absorption of organic acids including penicillin 2 from cerebrospinal fluid (Fishman, 1964) and $\overrightarrow{\vec{O}}$ from aqueous humour (Becker, 1960) and is 3 similar to that in the renal proximal tubule.

These experiments were designed to determine? whether active reabsorption of ampicillin from the lungs is a factor responsible for the low levels응 of the drug in sputum.

Evidence is presented in this paper suggesting that ampicillin is absorbed from the lungs by. passive diffusion rather than by active transport.

\section{SUBJECT AND METHODS}

These studies were performed on a 28-year-old healthyo male volunteer (J.L.M.). The subject had fasted overnight and before the intratracheal injection drank a pint of water to ensure that urine could be passed during the experiment.

INTRATRACHEAL INJECTION The subject was seated upright in a chair with the neck extended, and the skinfo overlying the cricothyroid membrane was infiltrated $\mathbb{\Phi}$ with $2 \%$ lignocaine. After a few minutes $2 \mathrm{ml}$ of $2 \% ?$ lignocaine was injected into the trachea through the $\square$ cricothyroid membrane. This was followed by vigorous coughing and resulted in numbing of the throat and some loss of voice. A few minutes later $\stackrel{P}{\mathbb{D}}$ a further $2 \mathrm{ml}$ of $2 \%$ lignocaine was injected into the trachea to test for abolition of the cough reflex. When 
this had occurred, $50 \mathrm{mg}$ of ampicillin dissolved in $5 \mathrm{ml}$ of sterile pyrogen-free water was injected into the trachea. Venous blood samples were taken after 20 and 40 minutes, $1,1 \frac{1}{2}, 2,2 \frac{1}{2}, 3,3 \frac{1}{2}$, and 4 hours and the plasma was separated. All urine passed $\frac{1}{2}, 1$, $1 \frac{1}{2}$, and 2 hours after the intratracheal dose was collected and the volume measured.

This procedure was repeated at weekly intervals using doses of $250 \mathrm{mg}$ and $1,250 \mathrm{mg}$ of ampicillin respectively.

The effect of probenecid on ampicillin absorption from the lungs was studied by giving ampicillin 250 $\mathrm{mg}$ together with probenecid $100 \mathrm{mg}$ in $5 \mathrm{ml}$ of water by intratracheal injection and taking venous blood samples as before.

BRONCHIAL SLICE TECHNIQUE Cross and Taggart's (1950) renal slice technique for the study of active transport of organic acids by the kidney was modified and used with bronchial slices. This method involved incubating slices of bronchus in Krebs-Ringer solution containing ampicillin and measuring its concentration in the tissue and culture medium after a period of incubation.

Human foetal bronchus was obtained from the Royal Marsden Hospital Organ Culture Bank, London.

Slices of bronchus less than $0.5 \mathrm{~mm}$ thick were cut with a razor, dried with blotting paper, and weighed. Bronchial slices weighing approximately $200 \mathrm{mg}$ were divided into four aliquots. Each aliquot was placed on a piece of filter paper $(2 \times 3 \mathrm{~cm})$ and floated on $5 \mathrm{ml}$ of culture medium (Krebs-Ringer solution containing $20 \mu \mathrm{g} / \mathrm{ml}$ of ampicillin) in a plastic Petri dish as shown in Figure 1. A stream of gas $\left(95 \% \mathrm{O}_{2}\right.$ and $\left.5 \% \mathrm{CO}_{2}\right)$ was passed into the culture medium beneath the filter paper by piercing the side of the Petri dish with a No. 1 needle. This stream of gas served to keep afloat the filter paper which tended to sink when wet, agitated and buffered the culture medium, and supplied oxygen to the slices. The bronchial slices were incubated at $37^{\circ} \mathrm{C}$ for 16 hours. After this period they were shown to be viable by the presence of active ciliary movement observed microscopically. Homogenization of the bronchial slices was performed with a glass tissue grinder, and samples of the supernatant together with samples of the culture medium were obtained.

All samples for ampicillin assay were stored at $-20^{\circ} \mathrm{C}$ until analysed. Ampicillin was assayed in the

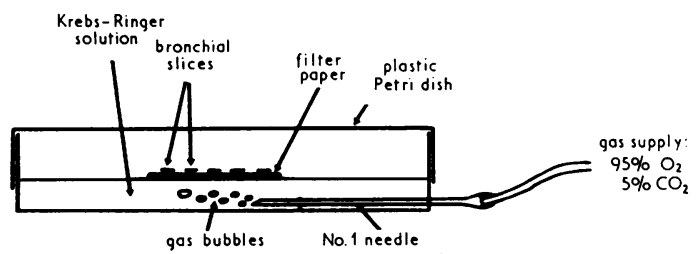

FIG. 1. Cross-sectional diagram of apparatus used for bronchial slice studies. samples, within two days of collection, by the cupplate method using Sarcina lutea as a test organism.

\section{RESULTS}

When ampicillin was injected into the trachea it provoked a desire to cough. After doses of $50 \mathrm{mg}$ and $250 \mathrm{mg}$ this was voluntarily suppressed, but after the $1,250 \mathrm{mg}$ dose this was not possible and cough with expectoration occurred; data obtained after this dose have therefore to be interpreted with caution.

Plasma levels of ampicillin obtained after various intratracheal doses are shown in Figure 2. These results show that the plasma levels of ampicillin increase with the intratracheal dose. Another measure of absorption used was the area under the plasma level-time curve. For the three doses the ratio of the areas under the plasma level-time curves was $1: 3.5: 23$, again showing that absorption of ampicillin increased with the intratracheal dose.

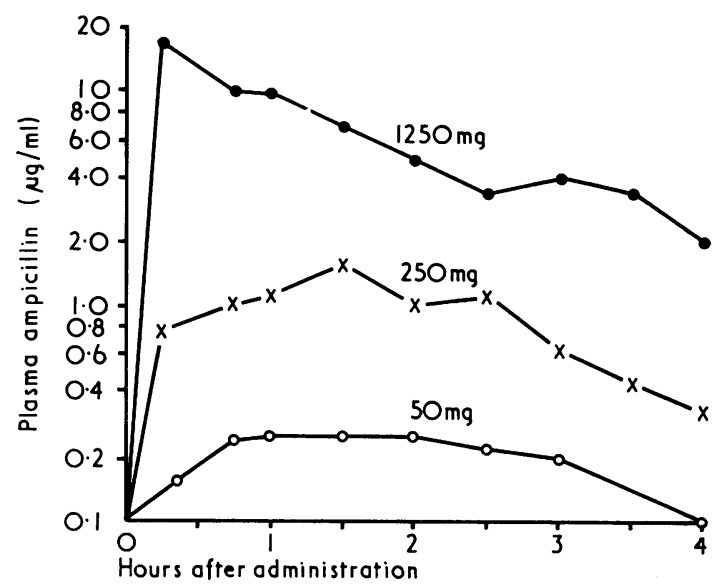

FIG. 2. Comparison of plasma levels of ampicillin after different intratracheal doses: $\bullet \longrightarrow 1,250 \mathrm{mg}$; $\mathrm{X}-\mathrm{X} 250 \mathrm{mg}$; $\multimap 50 \mathrm{mg}$.

As shown in the Table, the larger the intratracheal dose of ampicillin, the greater is the amount excreted in the urine. Ratios of the cumulative weights of ampicillin excreted in the urine after each of the three doses were as follows: $1: 7: 88$ (30 minutes); $1: 5: 48$ (1 hour); $1: 5: 42$ (1 $\frac{1}{2}$ hours); and 1:5:35 ( 2 hours), while the ratio of the doses was $1: 5: 25$. Considering the urinary excretion of ampicillin after the $50 \mathrm{mg}$ and $250 \mathrm{mg}$ doses only (there was coughing after the $1,250 \mathrm{mg}$ dose), after $1,1 \frac{1}{2}$, and 2 hours the ratio of the 
T A B L E

CUMULATIVE WEIGHT OF AMPICILLIN (mg) EXCRETED IN URINE AFTER VARIOUS INTRATRACHEAL DOSES

\begin{tabular}{c|ccc}
\hline \multirow{2}{*}{$\begin{array}{c}\text { Time after } \\
\text { Intratracheal } \\
\text { Dose (hr) }\end{array}$} & \multicolumn{3}{|c}{ Intratracheal Dose (mg) } \\
\cline { 2 - 4 } & 50 & 250 & 1,250 \\
\hline 0.5 & 1.2 & 8.9 & 105.6 \\
1 & 4.0 & 20.9 & 192.5 \\
1.5 & 5.8 & 30.5 & 242.9 \\
2 & 7.8 & 38.9 & 271.5 \\
\hline
\end{tabular}

cumulative weight of ampicillin excreted in the urine was $1: 5$, which is the ratio of the doses. This is the ratio one would expect to find if ampicillin was absorbed from the lungs by passive diffusion.

Coughing following the $1,250 \mathrm{mg}$ dose of ampicillin would tend to increase its distribution in the lungs and the high rate of absorption after this dose might be due to an increase in the lung surface exposed to this drug.

The effect of probenecid on the plasma levels of ampicillin after intratracheal administration is shown in Figure 3. Plasma levels of ampicillin after an intratracheal injection of ampicillin and

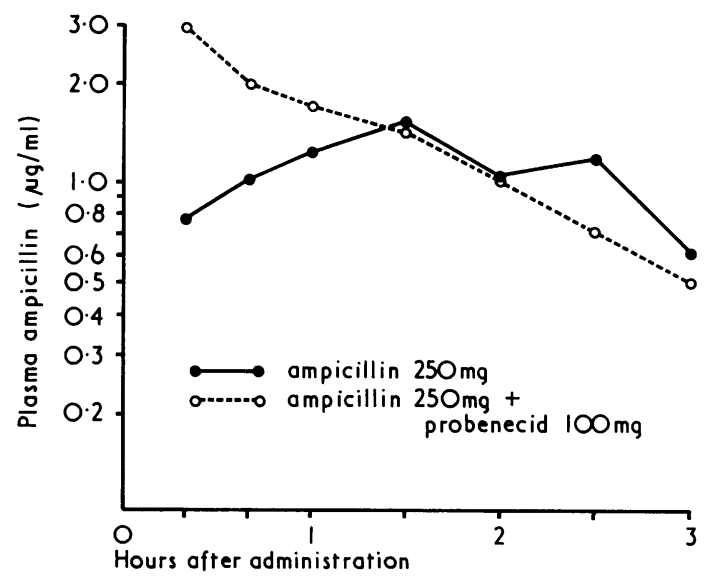

FIG. 3. Comparison of plasma levels of ampicillin after intratracheal injection of (a) ampicillin $250 \mathrm{mg}$ - and (b) ampicillin $250 \mathrm{mg}+$ probenecid $100 m g$ o.....

probenecid were initially higher than after ampicillin alone. The reason for this is not known. Nevertheless these results demonstrate lack of blockade of ampicillin absorption by probenecid.

A typical example of the uptake of ampicillin by human foetal bronchial slices is as follows:
Weight of bronchial slices $=193 \mathrm{mg}$

Weight of ampicillin in bronchial slices $=1.5 \mu$

Concentration of ampicillin in bronchias.

slices $=\frac{1 \cdot 5}{0 \cdot 193}=7 \cdot 8 \mu \mathrm{g} / \mathrm{g}$.

Concentration of ampicillin in medium है $8.0 \mu \mathrm{g} / \mathrm{ml}$

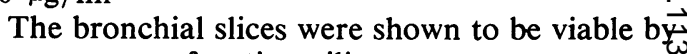
the presence of active ciliary movement.

These results show no evidence of accumulatio $\frac{\vec{P}}{\times}$ of ampicillin by human foetal bronchial slices.

\section{DISCUSSION}

Using plasma levels, the area under the plasmo level-time curve and urinary excretion as measures of ampicillin absorption from the lungs $\overrightarrow{{ }_{\pi}}$ the rate of absorption of the drug was found to increase with the intratracheal dose. There is no evidence of rate limitation of absorption as woul occur with saturation of an active transport mechanism for organic acids (Schanker, 1962) o and probenecid failed to reduce ampicillin absorp:tion from the lungs. These findings, although preo liminary, suggest that ampicillin is absorbed from the lungs by passive diffusion. Similar finding were obtained with acidic drugs such as $\mathrm{p} \frac{2}{\mathbb{Q}}$ aminohippurate in rats by Enna and Schankep (1970). They found that organic acids were ab호 sorbed from the lungs by passive diffusion aB rates related to their molecular size and lipid $E_{E}$ water partition coefficient.

From the present in vivo studies on pulmonary absorption of ampicillin it is not possible to distinguish between bronchial absorption and alveolar absorption, the results reflecting absorp $\frac{O}{3}$ tion from both surfaces. The results of the bronchial slice technique, however, provide nog evidence of an active transport mechanism for ampicillin in the human bronchus. Similar findings have also been obtained with human bronchus using para-aminohippurate (Maddocks, 1973)․ These results fail to confirm the suggestion of May and Delves (1965) that ampicillin might be actively secreted by the bronchial mucosa.

Thus it appears that the poor penetration of ampicillin into bronchial secretions is related to its physicochemical properties, particularly its strongly acidic group ( $\mathrm{pKa} 2.52$ ) and its poos solubility in lipids. This gives rise to the question whether the levels of ampicillin in bronchial secre $\frac{0}{0}$ tions may be increased by some means. There are़ two approaches to this problem. First, the perme ability of the blood-bronchial fluid barrier migh $\mathbb{R}$ be increased. Buergi, Regli, and Medici (1969 
reported that bromhexine increased the penetration of oxytetracycline into sputum but Ingold and Shaylor (1971) found it had little effect on the penetration of ampicillin into sputum. Secondly, the ampicillin molecule might be modified, provided there is no loss of antibacterial activity, by introducing a suitable functional group to make it a weaker acid (Albert, 1952). Also the ampicillin molecule might be chemically modified, the so-called drug latentiation of Harper (1959), so that the biologically active compound, ampicillin, is released in bronchial secretions.

I am indebted to the late Professor J. Robert May for his helpful advice. The Chest and Heart Foundation provided financial support.

\section{REFERENCES}

Albert, A. (1952). Ionization, $\mathrm{pH}$ and biological activity. Pharmacological Reviews, 4, 136.

Becker, B. (1960). The transport of organic anions by the rabbit eye. American Journal of Ophthalmology, 50, 862.

Buergi, H., Regli, J., and Medici, T. (1969). Observation on the problems accompanying antibiotic treatment in bronchitis with recurrent infection. Arzneimittel-Forschung, 19, 776.

Cross, R. J. and Taggart, J. V. (1950). Renal tubular transport: accumulation of p-aminohippurate by rabbit kidney slices. American Journal of Physiology, 161, 181.

Enna, S. J. and Schanker, L. S. (1970). Pulmonary absorption of organic compounds. Federation Proceedings, 29, 663.
Fishman, R. A. (1964) Active transport and the bloodbrain barrier to penicillin and related organic acids. Transactions of the American Neurological Association, 89, 51.

Goldman, J. N., Broughton, W., Javed, H., and Lauderdale, V. (1973). Ampicillin, erythromycin and chloramphenicol penetration into rabbit aqueous humor. Annals of Ophthalmology, 5, 147.

Harper. N. J. (1959). Drug latentiation. Journal of Medicinal and Pharmaceutical Chemistry, 1, 467.

Ingold, A. and Shaylor, J. M. (1971). The influence of bromhexine (Bisolvon) on the levels of ampicillin and oxytetracycline in sputum. British Journal of Diseases of the Chest, 65, 243.

Maddocks, J. L. (1973). Aspects of the chemotherapy of chronic bronchitis. M.D. thesis, University of London.

May, J. R. (1968). Chemotherapy of Chronic Bronchitis, 1st ed., p. 67. English Universities Press, London.

— and Delves, D. M. (1965). Treatment of chronic bronchitis with ampicillin: some pharmacological observations. Lancet, 1, 929.

Rapson, H. D. C. and Bird, A. E. (1963). Ionisation constants of some penicillins and of their alkaline and penicillinase hydrolysis products. Journal of Pharmacy and Pharmacology, 15, Supplement, p. 222.

Schanker, L. S. (1962). Passage of drugs across body membranes. Pharmacological Reviews, 14, 501.

Taber, L. H., Yow, M. D., and Nieberg, F. G. (1967). The penetration of broad spectrum antibiotics into the cerebrospinal fluid. Annals of the New York Academy of Sciences, 145, 473.

Requests for reprints to: Dr. J. L. Maddocks, KRUF Institute of Renal Disease, Cardiff Royal Infirmary, Cardiff. 